Research Report

\title{
Neurophysiological differences between perception and imagery
}

\author{
Harald T. Schupp ${ }^{\text {a,* }}$, Werner Lutzenberger ${ }^{\text {a }}$, Niels Birbaumer ${ }^{\text {a,b,* }}$, Wolfgang Miltner ${ }^{\text {a }}$, \\ Christoph Braun ${ }^{\mathrm{a}}$ \\ ${ }^{a}$ University of Tübingen, Institute of Medical Psychology and Behavioral Neurobiology, Tübingen, Germany \\ ${ }^{\mathrm{b}}$ Università degli Studi, Padova, Italy
}

Accepted 26 April 1994

\section{Abstract}

The present study was designed to examine brain activity underlying mental imagery. Since mental imagery is conceptualized as behavior guided by internal representation only, the activity of the prefrontal lobes was assumed to be a measure of differentiation of imagery from perception. Twenty-one subjects were requested to observe and imagine a swinging pendulum and to touch and imagine a coshball in separate trials. The EEG was recorded from 15 standard electrode sites and analyzed with (1) traditional alpha power and (2) an estimation of dimensional complexity (a.measure derived from nonlinear dynamics). Both EEG measures revealed expected object-related differences during perception as well as during imagery. The visual pendulum showed relative to the tactile coshball increased dimensional complexity and less alpha power at parietal and frontal sites. However, only the EEG dimension supported the main hypothesis: Imagery resulted in increased prefrontal dimensional complexity in comparison to perception independent of the modality of the image. In contrast, for alpha power the difference between imagery and perception was due to stimulus modality.

Keywords: Imagery; Dimension; Deterministic chaos; EEG; Perception

\section{Introduction}

One view of imagery has focused on the internal manipulation of sensory information. This approach stresses the quasi-pictorial nature of visual images $[19,29,45]$. It is widely believed that the internal constraints of our perceptual system reflect invariant constraints of our subjective world, and that the perceptual constraints also hold during imagery [44]. Another approach to mental imagery describes imagery as hypothetical, anticipatory or 'as if' behavior [43] and focuses more on the motor aspects of mental imagery [9]. Considering interconnected cell assemblies as the units of representation of perceptual and mental events and information [7], it seems reasonable to assume a uni-

\footnotetext{
* Corresponding authors. Institut für Medizinische Psychologie und Verhaltensneurobiologie, Universität Tübingen, Gartenstr. 29, D-72074 Tübingen, FRG. Fax: (49) 7071-295659; E-mail: sipbi@comserv1.zdv.uni-tuebingen.dbp.de.
}

tary view of sensory as well as motor images. The aim of the present study was to extend those approaches by emphasizing that imagery is guided by internal representations only, a process which was expected to result in specific brain activity underlying all imagery processes.

In a now-classic paper, Hebb [26] argued that sensory imaging constitutes the activation of higher-order (more 'abstract') cell assemblies also involved in the perception of the same events. Perception of a complex object activates cell assemblies along a dimension from sensory and concrete to abstract and conceptual. Thus, perception can be characterized as externally guided activation of concrete as well as 'abstract' assemblies. In contrast, imagery consists of the internal activation of predominantly 'abstract' cell assemblies, sometimes even without engagement of the concrete sensory cell assemblies. We assume that more abstract categorical representations involve more widespread and less focused brain activity while the concrete assemblies represent more localized brain responses at and near the primary cortical areas. 
Evidence for the involvement of the same higherorder cell assemblies in imagery as in perception comes from psychophysiological and neuropsychological studies. For example, Goldenberg et al. [23,24] compared visual imagery conditions with no-imagery control conditions. The imagery questions caused enhanced occipital blood flow in comparison to the nonimaginal questions. Davidson and Schwartz [10] found alpha attenuation over postcentral areas during imaging ones' forearms being tapped and alpha attenuation over visual areas during imaging a flashing light. In an event-related potential study [17], subjects imaged H's or T's while they performed a detection task with H's and T's. Match stimuli (e.g. imaging $\mathrm{H}$ and presenting $\mathrm{H}$ ) resulted in an enhanced occipital negativity of the first negative peak when compared to mismatch stimuli. Finally, neuropsychological studies revealed that specific perceptual deficits cause parallel deficits in the corresponding imagery ability. For example, deficits in color vision led to a loss of color imagery [16].

From another perspective, imagery can be seen as behavior guided only by internal representations or models without external stimuli [25]. Imagery requires an 'action plan' containing the temporal organization of the activation of the involved cortical networks and the inhibitory control of visible motor outflow [11]. The prefrontal cortex seems to be an essential structure for this kind of higher cognitive function [22,25]. There are several studies which revealed prefrontal activation during imagery $[12,28,41,42]$.

The evidence for prefrontal involvement in imagery comes exclusively from studies involving motor action in imagery. Roland and Friberg [42] measured the regional cerebral blod flow (rCBF) while subjects imaged a walk. The rCBF increased in an area-specific manner in anterior and posterior regions of the brain. The increases in the posterior regions of the brain, including the occipital lobe and parieto-temporal areas, revealed the activity of cell assemblies required for the retrieval of visual and spatial memory. The increases in prefrontal areas indicate an organizational function of the prefrontal cortex during imagery. Decety et al. [12] compared motor behavior and motor imagery more directly. Subjects were required to perform and image graphic gestures. Measuring rCBF, they concluded that motor behavior and motor images needed both the integrative activity of the prefrontal cortex as well as the supplementary motor areas and other areas involved in motor programming.

The primary goal of this study was to demonstrate the specific role of the prefrontal cortex for sensory images. A related purpose of this study was to analyze the EEG with a new methodology derived from nonlinear system theory. One of the measures used in this research to describe dynamical brain activity was dimensional complexity. It was shown that the nonlinear measure captures new information about the dynamic activity of the brain [31,32,46]. We hypothesized that imagery leads to a more 'complex' (i.e. less predictable) pattern of electrocortical activity at prefrontal sites.

\subsection{Dimensional complexity: a brief introduction}

Nonlinear dynamics offers new insights relevant for the understanding of brain function. The rapid augmentation of activity ('ignition') in cell assemblies is obviously a nonlinear phenomenon [7]. Even single neurons show characteristic nonlinear phenomena (review in Elbert et al. [13]). In contrast to linear systems, nonlinear systems can show irregular, chaotic behavior. The concept of deterministic chaos has demonstrated, that seemingly chaotic features can be governed by a few nonlinear differential equations. Thus, deterministic chaos refers to apparently lawless behavior ruled by (deterministic) laws [13]. Two prominent features of deterministic chaos are unpredictability over extended time periods, and sensitive dependence on initial conditions.

Although the differential equations for the brain's electrical activity are unknown, it is possible to reconstruct from a single EEG trace the phase-space attractor and to calculate the dimension of this reconstructed attractor. Chaotic attractors do not have an Euclidean integer dimension, but are described by a fractal or noninteger dimension.

Compared to EEG during the waking state, EEG from stage 2 sleep has a lower dimension, and stage 4's dimension is even lower [4]. REM sleep has a dimension comparable to the waking EEG [3]. A marked lowering of dimension was observed during epileptic seizures [2,27,37]. In addition, the dimension in the waking state is sensitive to processing load. Increased dimension is found during performance of mathematical calculations [35,39]. Lutzenberger et al. [31] found increased dimension in subjects with high IQ compared to subjects with low IQ but only during rest. During performance of a complex task, less intelligent people increased their dimension to a level similar to more intelligent subjects. Lutzenberger et al. [32] reported higher dimension during imagery than during various sensory tasks. However, since imagery contents were different from sensory non-imagery tasks a direct comparison of these two types of tasks was not possible.

Currently, various mathematical procedures are used to compute the fractal dimension, which give different results when applied to the same data set. Furthermore, calculation of the true fractal dimension requires extended time-series [47] which is in conflict with the non-stationarity of brain activity. Following Rapp et al. [39], relative differences in dimension between conditions are of more interest than the absolute fractal dimension. In our calculations [31,32], we combined 
the singular value decomposition introduced by Broomhead and King [8,1] with the method of averaged pointwise dimension developed by Farmer et al. [18] and referred to our measure as dimensional complexity ${ }^{1}$.

\subsection{Research problem}

We compared perception and imagery of a visuospatial object and a tactile object directly. We predicted the following pattern of brain activity: First, the prefrontal cortex was expected to reveal more complex activity and less pronounced frontal enhancement of alpha power during imagery than during perception for both the tactile and the visuo-spatial task. It was of major interest to look for modality-independent differences between imagery and perception. Second, we expected area-specific differences in content-specific cell assemblies. With respect to the tactile stimuli, the visuo-spatial object was expected to reveal more complex activity and less alpha power (i.e. more activation) at posterior regions. The somatomotor cortex and its association areas should be more activated by the tactile stimuli. In general, the comparison between imagery and perception was expected to reveal the activation of the same higher-order cell-assemblies, but imagery was expected to result in a more complex state of the relevant systems.

\section{Materials and methods}

\subsection{Subjects}

Twenty-two paid volunteers (10 female and 12 male) participated in the experiment. The ages ranged from

\footnotetext{
${ }^{1}$ A general discussion and caveats of the application of nonlinear dynamics to physiological signals is provided by Elbert et al. [13]. One issue under discussion relates to the point that dimensional complexity is not sufficient to differentiate stochastic or deterministic processes generating the time series under study. An additional test was the comparison of the dimensional complexity of the original EEG time series with the so-called filtered noise dimensional complexity: the Fourier-transformed EEG series were randomly phaseshifted and transformed back to the time domain [37]. Using our method of estimating dimensional complexity, we recently reported statistical evidence that the EEG cannot completely be described as filtered noise [32]. Further control analyses were conducted to investigate the influence of the length of the time series on the estimation of the dimensional complexity. Using the data set reported in the present study, we found that a systematic shrinking of the time series from $20.48 \mathrm{~s}$ down to $2 \mathrm{~s}$ resulted in a systematic underestimation of dimensional complexity but statistical differences between conditions remained stable. Thus, although the EEG is non-stationary and high-dimensional, the calculation of dimensional complexity is meaningful as a relative measure (between conditions).
}

18 to 35 . Subjects with a history of central nervous diseases or subjects currently on medication were excluded from the sample. The data from one man were lost due to computer failure.

\subsection{Design}

The present study consisted of two conditions, both including a single perception and imagery phase, but with different objects. In the pendulum condition subjects were required first to observe the swinging pendulum and then immediately after the perception phase to imagine the pendulum moving. In the other condition we used a coshball (a dandelion shaped piece of elastic rubber) as a tactile stimulus, which was handled with both hands in the perception phase and then imagined. All phases lasted for $45 \mathrm{~s}$. Half of the subjects started with the pendulum condition, the other half with the coshball condition. Subjects were instructed to keep their eyes open during all phases. During the imagery periods and the coshball handling subjects were instructed to keep eyes open and to avoid eye movements. The experimenter entered the room between the perception and imagery condition and removed the object. Instructions were given separately for both tasks in both written and verbal forms.

\subsection{Subjective ratings}

After each condition, subjects rated the vividness of their image for the pendulum and the coshball on a 9-point analog scale.

\subsection{Physiological recordings}

The EEG was recorded from 15 sites using an electrode placement cap with tin electrodes (ElectroCap International) according to the international 10-20 system and linked $\mathrm{Ag} / \mathrm{AgCl}$ electrodes placed on the mastoids. Electrical impedance was $5 \mathrm{k} \Omega$ or less for all electrodes. The electrodes were placed at the following placements: F7, F3, Fz, F4, F8, T3, C3, Cz, C4, T4, T5, P3, Pz, P4, T6. All channels were amplified with a bandwidth from 0.016 to $35 \mathrm{~Hz}$. All leads were referenced to the vertex electrode for recording purposes, off-line corrected for time lags in the sampling procedure [33], and changed to an average reference giving all electrodes equal weight. All data were sampled at a rate of $100 \mathrm{~Hz}$. Vertical eye movements were measured at the right eye using the Fp1 (EEG) electrode and a miniature Beckman $\mathrm{Ag} / \mathrm{AgCL}$ electrode below the right eye. The EEG was corrected for ocular artifacts with a regression method based upon the vertical and horizontal EOG [15]. For each condition an interval of $20.48 \mathrm{~s}$ was selected for the computation of the EEG measures. The interval was centered around the mid of 
the whole $45 \mathrm{~s}$ periods, thereby avoiding non-stationary EEG shifts due to the beginning of each perceptual or imaginal phase.

\subsection{Data analysis}

Alpha power and dimensional complexity were calculated for every EEG trace. The resulting values were submitted to an ANOVA to search for EEG differences related to the experimental conditions.

\subsection{Alpha power}

The EEG alpha power was obtained from the average log power in the range from $8-12 \mathrm{~Hz}$. The power spectrum was calculated by averaging the Fourier transforms of 15 overlapping 2,56-sec segments (256 points), using Parzen windows.

\subsection{Dimensional complexity}

The dimensional complexity was calculated for every recording site and condition separately. The algorithm consists of two principal steps: The first step is to seperate the signal from the noise of the time series and to determine the embedding dimension used to reconstruct the state space by means of a singular. value decomposition, SVD (see Fig. 1 A-C). The second step (see Fig. 1D,E) is to estimate the dimensional complexity. The steps of the calculation are illustrated in Fig. 1 by applying our algorithm to a synthetic amplitude-modulated sine wave. (A) The first step is based on the autocovariance function. The covariances of the EEG-trace with time-shifted copies of the same signal were calculated. Time lags were varied from 0 to 31 time points, resulting in a symmetrical $32 \times 32$ matrix with the covariances as elements. The first row was the autocovariation function itself, in the second row, elements were shifted by one column to the right, in the third row by two columns etc. so that the diagonal element was always the covariance with time lag zero.

(B) The autocovariance matrix is then orthogonally rotated to a new orientation by means of a singular value decomposition (SVD [1,8], see also Fraser [20] for more information and critics) which is a noise reduction technique, related to principal component analysis or factor analysis. Each row of the rotated autocovariance matrix comprises a basic vector. The variance each vector accounts for is determined by its eigenvalue. A basic feature of the SVD is that the vectors are ordered according to the amount of variance each vector accounts for. Thus, the rotation results in 32 orthogonal components each of which can be considered as a dimension of the state space; they include the signal and the noise. The size of the embedding dimension can be effectively reduced by

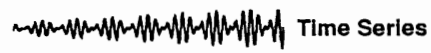

A
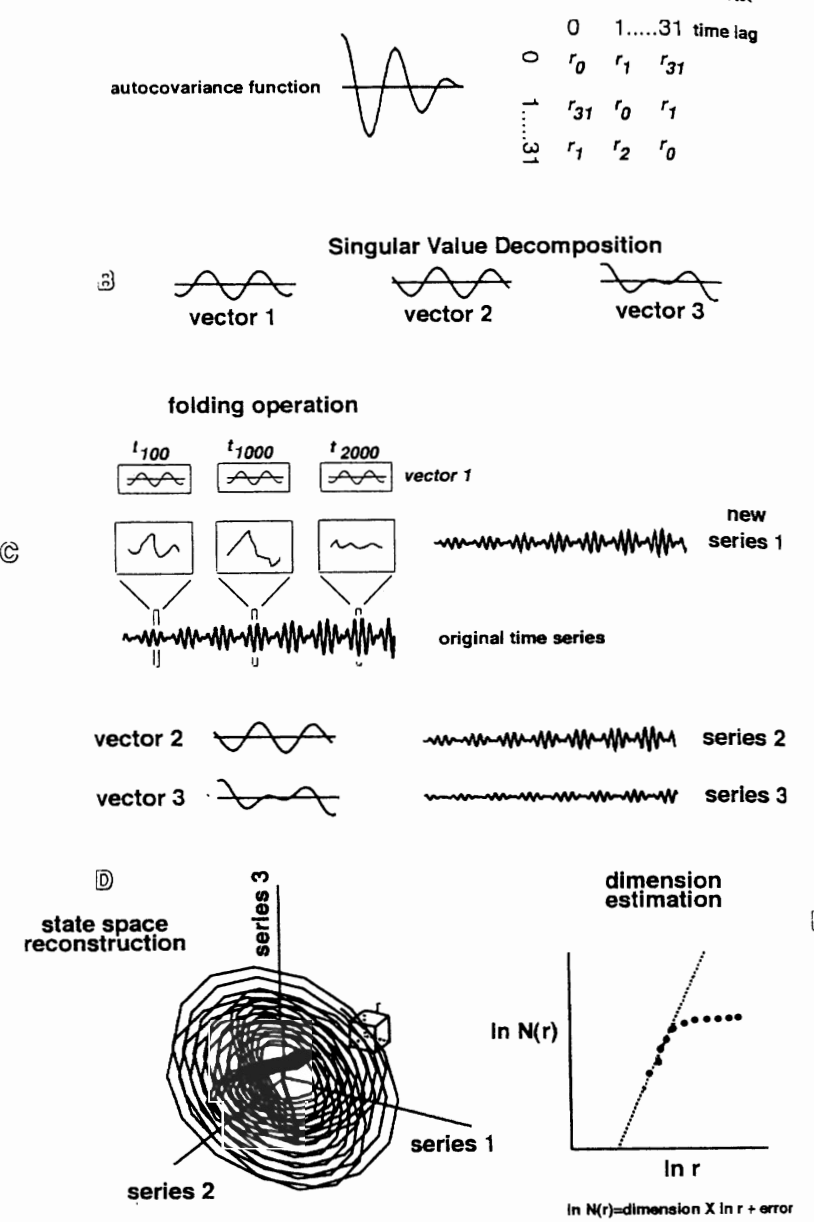

Fig. 1. Illustration of the major steps used for the calculation of dimensional complexity (for explanation see text).

discarding the components that account for little variance since they probably represent noise. In order to separate noise as well as activity with little variance from the signal a cut-off criteria for the number of accepted dimensions was chosen such that only vectors with eigenvalues larger than twice the smallest of all 32 vectors were selected. The criterion may seem somewhat arbitrary but it has two advantages: It is independent of the particular gains used and it is reproducible in different laboratories. In the present example, as illustrated in Fig. 1B, three vectors out of 32 vectors were considered as representing the signal and the remaining were discarded as negligible noise according to our cut-off criterion. Applying our algorithm to the analysis of the human EEG, the SVD typically extracts 15 vectors. Thus, the net result of the SVD applied to the autocovariance matrix is a small set of time series $(\ll 32)$ whose sum closely approximates the 
original EEG time series ${ }^{2}$. (C and D) The extracted vectors were used to reconstruct the state space, with the number of extracted vectors determining the dimensionality of the state space. A folding operation of extracted vector and original time series was used to calculate a new and separate time series for each extracted vector. The calculation of the new time series for the first vector is illustrated in Fig. 1C. The first value of the new time series is the cross product of a 32 points segment of the original time series beginning at $t_{\mathrm{o}}$ and the vector 1 . The next value $\left(t_{1}\right)$ is the cross product computed between the vector and a 32 points segment of the original time series beginning at $t_{1}$, and so forth ${ }^{3}$.

The calculated time series (one for each vector) are used for the state space reconstruction. Thus, as shown for the example (see Fig. 1D), each point is now exactly localized in the three-dimensional state space according to its value on each of the three time series resulting in the shown trajectorie. As mentioned above, the SVD applied to the human EEG typically extracts 15 dimensions, and therefore the human EEG is usually placed in a 15-dimensional state space.

(D and E) The method of pointwise dimension [18] was then applied to calculate the dimensionality of the subspace occupied by the trajectorie ${ }^{4}$. Given a distinct reference point, the number of points $N(r)$ which lie in a hypercube with radius $r$ around this chosen point is counted (see Fig. 1D). This counting is performed for subsequently larger radii until ultimately all points of the time series lie within this hypercube. The increase of the number of points lying in subsequently larger hypercubes, is used to determine the dimensionality of the state space. For Euclidean dimension, a one-dimensional line results in an increase of the number of data points with increasing radius $\left(r^{1}\right)$, for a two-dimensional plane, the number will increase with the square of the radius $\left(r^{2}\right)$. Generally, the number of

\footnotetext{
${ }^{2}$ The SVD was chosen not only in order to separate signal from noise but also as a method to develop a fully automatic procedure for the calculation of the dimensional complexity. Thus, the determination of delay time (or time lagging) is another implicit feature of the SVD in the current application. A variety of methods for the determination of the delay time were proposed such as first zero crossing of the autocorrelation or the first minimum of the mutual information. So far, there is no optimal procedure and the SVD has the advantage of determing delay time for every case independent of the experimenters.

${ }^{3}$ The new time series consists of 2016 time points only, because the folding procedure always requires 32 time points of the original time series.

${ }^{4}$ It is important to distinguish between the dimension of the state space which has in dissipative systems greater dimensions than the calculated dimensional complexity which refers to a subspace of the state space after the system has settled on this subspace.
}

data points will increase with $r^{d}$, where $d$ denotes the dimensionality of the attractor. In case of fractal dimensions, $d$ is a non-integer value. Plotting the number of data points against the radius on a double-logarithmic scale, dimension shows up as the the slope of the resulting curve.

For the present computations, 20 different radii $r_{i}$ were chosen. The distance between subsequent radii was selected such that each enlargement of the radius increased the total count by an equal number of points, i.e. $N\left(r_{i}\right)-N\left(r_{i}-1\right)=2016 / 20$. The counts are plotted against the radii using a double logarithmic scale (see Fig. 1E). The resulting function starts with a straight line of a certain slope, but then declines parallel to the abscissa. A linear fit is performed on the straight segment, its slope is used for further calculation of the dimensional complexity. In order to obtain an estimation of the straight segment, only the lowest ten values are chosen first. If the highest of these ten values has the largest distance to the straight line, the linear fit is recalculated for nine values only, because it is assumed that the tenth point is not belonging to the linear segment. If again the highest of these has the largest distance from the straight line, it is omitted from the next calculation. The process is repeated until the highest point has no longer the largest distance from the estimated straight line. Typically, this procedure results in a linear fit using the first five to seven lowest radii for the slope determination.

The last calculation step was repeated for 32 equidistant reference points and the slope was determined for each of the reference points. The median of the slope values was taken as the measure of dimensional complexity for the EEG time series. The dimensional complexity calculated this way in the awake adult results in values between 4 and 6 . In the present study, the mean dimensional value was 4.6 averaged across all conditions.

\subsection{Analysis of variance}

The resulting EEG values (one for complexity and one for alpha power) separated by condition and electrode were entered into an ANOVA with the following factors: (1) The repeated measure factor 'Object' with two levels: pendulum and ball. (2) Another repeated measure factor 'Image-Percept' contained two levels: Perception and Imagery. (3) The third factor 'Electrode' included the 15 electrode sites.

Expanded ANOVA's were calculated to reveal topographical information. For that purpose, the electrode sites were divided in three coronal rows and five sagittal rows. The factor 'Anterior-Posterior' included three levels: frontal (F7, F3, Fz, F4, F8), central (T3, C3, Cz, C4, T4) and parietal (T5, P3, Pz, P4, T6). The factor 'Laterality' included five levels: far left (F7, T3, 
T5), mid-left (F3, C3, P3), midline (Fz, Cz, Pz), midright (F4, C4, P4) and far-right (F8, T4, T6).

For illustrative purposes, brain maps for dimensional complexity and alpha power were calculated using the Akima smooth surface fit.

All ANOVAs were repeated measures ANOVAs having factors with levels exceeding two. Thus, for all ANOVA's Greenhouse-Geisser epsilon values were applied to correct for the lack of sphericities in the covariance matrices. Corrected $P$-values are reported throughout. Contrasts of groups of means were used as post-hoc tests.

\section{Results}

\subsection{Subjective ratings}

The reported vividness was higher for the coshball (mean of 6.4) in comparison to the pendulum (mean of $4.4)$ as indicated by a significant 'vividness' effect $\left(F_{1,20}\right.$ $=7.7, P=0.012)$. 16 out of the 21 subjects indicated higher vividness for the coshball than the pendulum.

Image - Percept
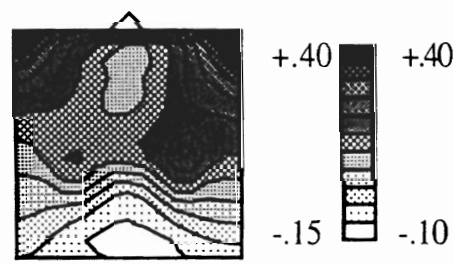

Pendulum - Coshball

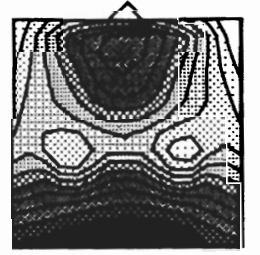

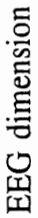
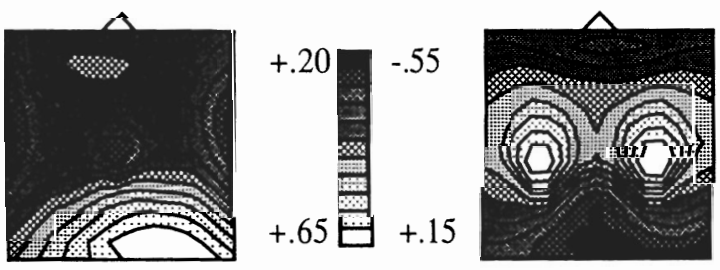

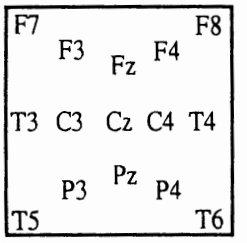

Fig. 2. The left side shows the difference maps (image minus percept) across stimuli modalities for dimensional complexity (top) and alpha power (bottom). The right side shows the difference maps (pendulum minus coshball) across task conditions (i.e. percept and image) for dimensional complexity (top) and alpha power (bottom). All maps represent grand average difference maps.
Pendulum - Ball
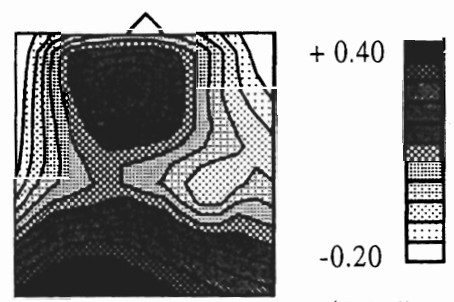

0.40

EEG dimension

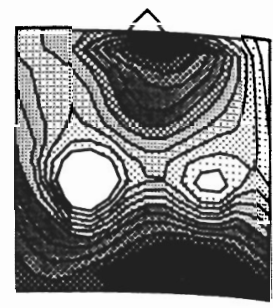

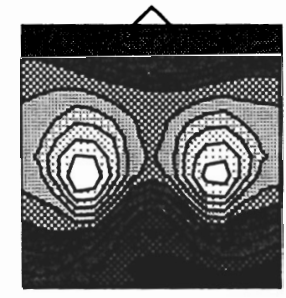

Percept

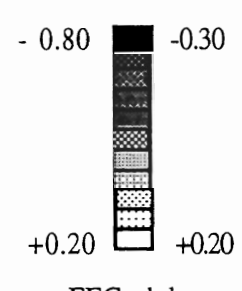

EEG alpha

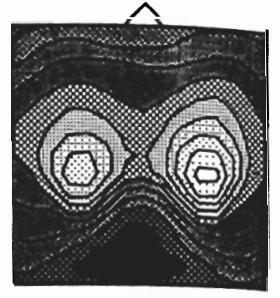

Image
Fig. 3. The difference maps (pendulum minus coshball) were calculated separately for (percept and image) condition and EEG measure (dimensional complexity and alpha power). Electrode positions are the same as in Fig. 2. Again, all maps represent grand average difference maps.

\subsection{Dimensional complexity}

Figs. 2 and 3 shows difference brain maps summarizing the dimensional complexity results. The top panel of Fig. 2 on the left side shows the difference map (image minus percept) across conditions (pendulum and coshball). This difference map illustrates the enhanced brain complexity necessary for imagery. The map indicates the highest differences in dimensional complexity at prefrontal areas, especially at the F7 and F8 electrodes but also at F3 and F4, and supports the hypothesis of additional frontal and prefrontal involvement during imagery.

The difference map (pendulum minus coshball) across conditions (at the top of the right side of Fig. 2) illustrates also the area-specific distribution of dimensionality. The brain map shows higher dimensionality at parietal sites and somewhat decreased dimensionality at central sites during the pendulum conditions compared to the coshball conditions. The higher involvement of frontal sites during the pendulum may be related to time estimation or ideomotor processes in the pendulum condition.

Fig. 3 (top row) shows the difference maps (pendulum minus coshball) separately for percept and image. The topographic patterns obtained for percept and image are quite similar, which supports the hypothesis that the same higher-order neural networks are in- 
Table 1

ANOVA results for dimensional complexity and alpha power

\begin{tabular}{llllll}
\hline & \multicolumn{2}{l}{ Dimension } & & Alpha power & \\
\cline { 5 - 6 } \cline { 5 - 6 } & $F$-value & $P$-value & & $F$-value & $P$-value \\
\hline Object & 2.5 & 0.1298 & 10.6 & 0.0039 \\
Percept-Imagery & 5.2 & 0.0333 & 13.9 & 0.0013 \\
Electrode & 6.5 & 0.0001 & 22.7 & 0.0001 \\
Object * Percept-Imagery & 1.2 & 0.2782 & 2.9 & 0.1040 \\
Object * Electrode & 4.4 & 0.0008 & 13.4 & 0.0001 \\
Percept-Imagery * Electrode & 7.5 & 0.0001 & 7.9 & 0.0001 \\
Object * Percept-Imagery * Electrode & 1.4 & 0.2213 & 4.8 & 0.0003 \\
\hline
\end{tabular}

volved during imagery as during perception. The principal difference between the percept and image conditions is the above-mentioned additional frontal involvement during imagery, which supports the hypothesis of increased dimensional complexity during imagery.

Table 1 shows the results of the three-way ANOVA. The highly significant results obtained with the 'Electrode' main effect and interactions involving this factor suggested further analysis using a four-factorial ANOVA to detect topographic differences. The 'Image-Percept' main effect was significant $\left(F_{1,20}=5.2\right.$, $P=0.03$ ), with imaging showing higher dimensionality than perception. Interactions involving this factor also occurred: 'Image-Percept $\times$ Anterior-Posterior' $\left(F_{2,40}\right.$ $=19, \quad P<0.0001)$ and 'Image-Percept $\times$ Laterality' $\left.F_{4,80}=3.1, \quad P<0.05\right)$. Post-hoc tests revealed higher dimensionality during imagery at the frontal row ( $F=$ 43.6, $P<0.0001)$ and also an increase at the central row $(F=11.2, P<0.01)$. In contrast, the dimensionality was reduced at the parietal rows $(F=4.8, P<0.05)$. The differences between imagery and perception were confined to the mid-lateral and lateral sites of both hemispheres. No differences between imagery and perception were found in the midline row, but higher dimensionality was obtained at the mid-left and midright rows (both $F$ 's $>4, P<0.05$ ) and were much more pronounced at the left and right rows (both $F$ 's $>20, \quad P<0.001)$. The three-way interaction 'Image-Percept $\times$ Anterior-Posterior $\times$ Laterality' $\left(F_{8,160}=3.1, P=0.01\right)$ is mainly due to the F7 and F8 electrodes which had low dimensionality during perception but strongly increased dimensionality during imagery (both $F$ 's $>45$ ).

Topographic differences between pendulum and coshball were indicated by interactions of 'Object $\times$ Anterior-Posterior' $\left(F_{2,40}=8.4, P<0.001\right)$ and 'Object $\times$ Anterior-Posterior $\times$ Laterality' $\left(F_{8,160}=2.3, \quad P<\right.$ $0.05)$. The only difference in dimensional complexity along the Anterior-Posterior dimension was a highly significant increased dimensionality at the parietal row for the pendulum in comparison to the coshball $(F=29$, $P<0.001)$. The three-way interaction 'Object $\times$ Anterior-Posterior $\times$ Laterality' arises mainly at the frontal row. The pendulum had higher dimensional complexity at F3, Fz and F4, significant at the F3 and $\mathrm{Fz}$ electrode $(F$ 's $>5, P<0.05)$, whereas the dimensionality was reduced at $\mathrm{F} 8(F=5.8, P<0.05)$ and non-significantly at F7 in comparison to the coshball.

For descriptive purposes, the coshball and pendulum data were submitted seperately to an ANOVA using the factors 'Image-Percept' and 'Anterior-Posterior' in order to test whether the frontal increase of the dimensionality was significant independently for both objects. The pendulum task revealed a significant main effect 'Image-Percept' $\left(F_{1,20}=5.8, \quad P=0.025\right)$, and a significant interaction 'Image-Percept $X$ Anterior-Posterior' $\left(F_{2,40}=14.8, P<0.0001\right)$. The imagery condition revealed increased dimensionality at frontal $(F=42, P<0.001)$ and central $(F=12, P<$ $0.01)$ sites compare to the perception condition. The coshball task also revealed a significant 'Image-Percept $\times$ Anterior-Posterior' $\left(F_{2,40}=8.6, P<0.0001\right)$ interaction. The dimensionality during imagery as compared to perception was increased at frontal sites $(F=12.3$, $P<0.001)$ and reduced at parietal $(F=5, P<0.05)$ sites. Thus, a significant increase of dimensional complexity during imagery was revealed at frontal sites by both objects.

\subsection{Alpha power}

Difference maps were calculated for the alpha power ${ }^{5}$ in the same way as for the dimensional complexity (see Figs. 2 and 3).

The three-way ANOVA revealed that all but one of the effects were significant (see Table 1), therefore we calculated the expanded four-way ANOVA ${ }^{6}$. Percep-

\footnotetext{
${ }^{5}$ The alpha power results are reported with regard to an average reference. The same analysis was also done with a linked-ears reference. The major difference between the two types of reference was the generally increased alpha power for the linked-ears reference. However, the qualitative differences reported in this section concerning the comparison percept-image and pendulum-coshball were obtained with both references.
} 
tion has lower alpha power than imagery (main effect 'Image-Percept'; $F_{1,20}=13.8, P<0.01$ ). The significant 'Image-Percept $\times$ Anterior-Posterior' $\left(F_{2,40}=19, P<\right.$ $0.0001)$ interaction demonstrated that although all three coronal rows had significantly increased alpha power during imagery, this was much more pronounced at the parietal row $(F=218)$ than at the central or frontal rows (both $F$ 's $<100$ ). The increases in alpha power during imagery were more pronounced at mid, mid-left and mid-right sagittal rows (all $F$ 's $>200$; 'Image-Percept $\times$ Laterality', $\left.F_{4,80}=4.6, P<0.01\right)$.

Overall, the coshball showed higher alpha power than the pendulum $\left(F_{1,20}=10,6, P<0.01\right)$. A significant effect 'Object $\times$ Anterio-Posterior' $\left(F_{2,40}=8.4, P\right.$ $<0.001)$ was found, with higher alpha power for the coshball at frontal $(F=39.3)$ and parietal $(F=104.5)$ rows. The 'Object $\times$ Laterality', $\left(F_{4,80}=6.1, P=0.002\right)$ interaction was also significant. The right, mid and left sagittal rows (all $F$ 's $>84$ ) revealed more pronounced higher alpha power of the coshball than the mid-left and mid-right rows ( $F$ 's $>32$ ). The interaction 'Object $\times$ Anterior-Posterior $\times$ Laterality' $\left(F_{8,160}=3.76, \quad P<\right.$ $0.01)$ is due to the central row which showed increased alpha power at T3, Cz, T4 $(F$ 's $>5.2, P<0.05)$ but significantly lower alpha power at $\mathrm{C} 3$ and $\mathrm{C} 4$ (both $F$ 's $>9.6, P<0.01$ ) for the coshball.

In contrast to the dimensional complexity data, the interactions' 'Object $\times$ Image-Percept $\times$ AnteriorPosterior' $\left(F_{2,40}=8.3, P=0.004\right)$ and 'Object $\times$ ImagePercept $\times$ Anterior-Posterior $\times$ Laterality' $\left(F_{8,160}=2.4\right.$, $P=0.05)$ were also significant. These interactions were followed up by analyzing the data for coshball and pendulum seperately with an ANOVA containing the factors 'Image-Percept', 'Anterior-Posterior' and 'Laterality'. For the coshball, the main effect 'ImagePercept' $\left(F_{1,20}=7.3, P=0.025\right)$ indicated a general increase of alpha power during imagery compared to perception. The interaction 'Image-Percept $\times$ Laterality' $\left(F_{4,80}=4.7, P<0.01\right)$ indicates that the differences between imagery and perception were more pronounced at the mid-left and mid-right rows. For the

\footnotetext{
${ }^{6}$ The reported statistical differences obtained for the perceptimage manipulation could be due to subtle variations on the eyesclosed or eyes-open dimension or due to a defocusing of the eyes in order to maintain images while the eyes are open. Thus, subjects were divided into two groups according their alpha power (high vs. low power in the alpha band) averaged over all conditions. An ANOVA that included this new group factor revealed that the significant differences of alpha power reported for the whole group in this section are entirely due to the group with high power in the alpha band. However, no relation was found between reported vividness and the level of alpha power. Also, after adding the group factor to the dimensional complexity analysis, there was no significant main effect or interaction containing the group factor. Thus, the suggested confound are relevant only for the alpha power analysis. More systematic studies are necessary to follow up this phenomenon.
}

pendulum, the main effect 'Image-Percept' $\left(F_{1,20}=10\right.$ $P<0.01)$ and the interaction 'Image-Percept $x$ Anterior-Posterior' $\left(F_{2,40}=16.1, \quad P<0.0001\right)$ were highly significant. Alpha power was increased dramatically during imagery at parietal $(F=158)$ sites but also enhanced at central $(F=21)$ and frontal $(F=64)$ rows. The 'Image-Percept $\times$ Laterality' $\left(F_{4,80}=3.2, P<0.05\right)$ interaction indicates more prounced alpha power increases at mid-right and right sites during imagery.

\section{Discussion}

The present study demonstrates for the first time that prefrontal EEG 'complexity' is increased during sensory imagery compared to the real perception of the same object. This confirms the hypothesis that during imagery there is a more complex pattern of electrocortical activity at prefrontal sites indicating the involvement of more independent processes generating the electrocortical activity. Alpha power was increased during imagery over the whole scalp in comparison to perception. However, as Fig. 2 revealed, this increase was much more pronounced in the posterior region, indicating less enhancement of fronto-central alpha power during imagery than perception.

The visual pendulum and coshball evoked different topographic patterns of brain activity. The pendulum showed a strong parietal increase in dimensionality in comparison to the tactile coshball and to a lesser extent also at frontal sites. The same regional differences in activation were found for alpha power too. In addition, imagining and perceiving the coshball resulted in activation (less alpha power) at C3 and C4, the projection areas of the fingers and hands (see. Fig. 2). Fig. 3 revealed similar topographic patterns for the difference map (pendulum minus coshball) for the perception and imagery conditions. Our results replicated earlier findings [10], thereby supporting Hebb's assumption that the same higher-order cell assemblies that are involved in perception are also relevant for imagery. In addition, frontally located working memory structures necessary for the delayed recall of the percept during imagery are implicated [25].

\subsection{Differences and similarities between dimensional complexity and Alpha power}

Initial observations [3] comparing dimensional complexity and alpha power suggest an inverse relationship between both measures. Lutzenberger et al. [32] and Pritchard and Duke [38] compared both measures more systematically. The alpha power is the frequency band which correlates better with dimensional complexity than beta or theta power, but a large fraction of the variance in dimensional complexity could not be ex- 
plained by alpha power (about 61\% in the Pritchard and Duke study). Furthermore, alpha power and dimensional complexity are sometimes different in their sensitivity to experimental conditions and variations in psychiatric groups, but behave in some conditions in parallel $[14,32]$.

In the present study, dimensional complexity and alpha power revealed expected findings but nonetheless important differences between both measures were found: First, the difference map (image minus percept) revealed a more localized frontal increase of dimensional complexity during imagery, whereas alpha power showed fronto-central activity. Second, the sensitivity of alpha power to manipulation of attentional focus (either external stimulation or internal processing) is dependent upon the stimulus modality (seè also Ray and Cole [40]). Perception of a visual stimulus depressed alpha power uniformly over the scalp. This reflects the intimate relationship between alpha power and visual attentional processes [34]. In contrast, all other conditions showed a parietal maximum, frontal medium and central minimum for alpha power. The increased alpha power during imagery confirms earlier reports [10] reflecting the different influences of the oculomotor system in imagery compared to focused visual attention during perception of the objects. Dimensional complexity doesn't seem to be sensitive to this variable but could indicate changes in intra- or inter-assembly interactions (see below). Overall, it is obvious that the dimensional complexity measure depicts information not available with traditional alpha power. The more localized increase of brain activity complexity at frontal sites, especially at F7 and F8 but also at F3 and F4, is in accordance with earlier findings from rCBF studies $[12,42]$.

\subsection{Cell assemblies and the concept of dimensional com- plexity}

The concept of cell assemblies is an important bridge between the microlevel of individual neurons and the macrolevel of mental activity [36] (e.g., thoughts, percepts, and images). Cortical cell assemblies formed between pyramidal cells with excitatory connections have the ability to ignite as a whole above their pre-excitation level when parts of the unit are activated. The classical way to study the ignition of cell assemblies is via external stimuli. It is assumed that the information flow goes from primary to higher-order cell assemblies [26]. In contrast, imagery as one form of internally guided behavior activates higher-order cell assemblies without external stimulation. The prefrontal cortex seems to be the key structure for this kind of cognitive function. The prefrontal cortex has extensive bidirectional cortico-cortical connections to associative areas [48]. It is also well connected to limbic areas, and has less extensive connections to primary projection regions. Thus, the connections of the prefrontal cortex are in accordance with the presumed function of this area. Current theories assume that imagery is the activation of interrelated associative cell assemblies containing stimulus, response and meaning elements $[5,6,9,30]$. Our results extend those theories, emphasizing the role of the frontal lobe in the generation and/or maintenance of the neural activity underlying imagery. Whether the functions of the prefrontal cortices during imagery consist of an inhibition or delay of motor execution or the generation and maintenance of a short term memory of the 'picture in the head' or both remains to be seen.

The present study, as well as other recent studies from our lab [14,31,32] and other groups [38] using group designs and multi-channel recordings, support the view that dimensional complexity as a relative measure between conditions within subjects leads to interpretable and consistent results. Increased dimensional complexity over a certain brain region may indicate a less ordered activation of associative 'nodes' in the upper cortical layers or as Freeman [21] put it, may indicate a more vigorous 'competition between cell assemblies' particularly in situations in which external stimulation has ceased to focus cortical processing at primary and secondary projection areas. An increased dimensional complexity might result from the less synchronously activation of parts of an assembly or different assemblies representing a specific object. This does not necessarily covary with the overall excitation or activation of the particular assemblies. Whatever the neurophysiological basis of dimensional complexity of electrocortical activity may be, mathematically an increasd dimensional complexity prefrontally indicates the contribution of a large pool of prefrontal neurophysiological processes during imagery.

\section{Acknowledgements}

We would like to thank the three anonymous reviewers for their suggestions, particularly for stimulating additional data analysis. The authors wish to thank Dr. Bruce Cuthbert and Dr. L. Bourne for their useful comments and suggestions for the manuscript and Gabi Walker for data collection. This Research was supported by the Deutsche Forschungsgemeinschaft (SFB 307).

\section{References}

[1] Albano, A.M., Mees, A.I., Guzman, G.C. and Rapp, P.E., Data requirements for reliable estimation of correlation dimensions. In H. Degn, A.V. Holden and L.F. Olsen (Eds.), Chaos in Biological Systems, Plenum, New York, 1987, pp. 207-220.

[2] Babloyantz, A. and Destexhe, A., Low dimensional chaos in an 
instance of epilepsy, Proc. Natl. Acad. Sci. USA, 83 (1986) 3513-3517.

[3] Babloyantz, A. and Destexhe, A., The Creutzfeld-Jacob disease in the hierarchy of chaotic attractors. In M. Markus, S. Mueller and G. Nicolis (Eds.), From Chemical to Biological Organization, Springer, Berlin, 1988.

[4] Babloyantz, A., Salazar, J.M. and Nicolis, C., Evidence of chaotic dynamics of brain activity during the sleep cycle, Phys. Lett., 111A (1985) 152-156.

[5] Birbaumer, N., Lang, P., Cook, E., Elbert, T., Lutzenberger, W. and Rockstroh, B., Self-regulation of slow brain potentials and imagery, Int. J. Neurosci., 39 (1988) 101-116.

[6] Birbaumer, N., Lutzenberger, W., Flor, H., Elbert, T. and Rockstroh, B., Imagery and brain processes. In N. Birbaumer and A. Oehman (Eds.), The Organization of Emotions, Hogrefe, Bern, 1994, pp. 122-138.

[7] Braitenberg, V. and Schüz, A., Anatomy of the Cortex, Springer, Berlin, 1991.

[8] Broomhead, D.S. and King, G.P., Extracting qualitative dynamics from experimental data, Physica, 20D (1986) 217-236.

[9] Cuthbert, B.N., Vrana, S.R. and Bradley, M.M., Imagery: function and physiology. In R.J. Jennings, P.A. Ackles and M.G.H. Coles (Eds.), Advances in Psychophysiology, Jessica Kingsley, London, 1991, pp. 1-42.

[10] Davidson, R.J. and Schwartz, G.E., Brain mechanism subserving self-generated imagery: electrophysiological specifity and patterning, Psychophysiology, 14 (1977) 598-601.

[11] Decety, J. and Ingvar, D.H., Brain structures participating in mental simulation of motor behavior: a neurophysiological interpretation, Acta Psychol., 73 (1990) 13-34.

[12] Decety, J., Philippon, B. and Ingvar, D.H., rCBF landscapes during motor performance and motor ideation of a graphic gesture, Eur. Arch. Psychiatry Neurol. Sci., 1988 (1988) 33-38.

[13] Elbert, T., Ray, W.J., Kowalik, Z.J., Skinner, J.E., Graf, K.E. and Birbaumer, N., Chaos and physiology, Physiol. Rev., 74 (1994) 1-47.

[14] Elbert, T., Lutzenberger, W., Rockstroh, B., Berg, P. and Cohen, R., Physical aspects of the EEG in schizophrenics, Biol. Psychiatry, 32 (1992) 595-606.

[15] Elbert, T., Lutzenberger, W., Rockstroh, B. and Birbaumer, N., Removal of ocular artifacts from the EEG - a biophysical approach to the EOG, Electroencephalogr. clin. Neurophysiol., 60 (1985) 455-463.

[16] Farah, M.J., Is visual imagery really visual? Overlooked evidence from neuropsychology, Psychol. Rev., 95 (1988) 307-317.

[17] Farah, M.J., Peronnet, F., Gonon, M.A. and Giard, M.H., Electrophysiological evidence for a shared representational medium for visual images and visual percepts, J. Exp. Psychol. General, 117 (1988) 248-257.

[18] Farmer, J.D., Ott, E. and York, J.A., Dimension of chaotic attractors, Physica, 7D (1983) 153-180.

[19] Finke, R.A., Theories relating mental imagery to perception, Psychol. Bull., 98 (1985) 236-259.

[20] Fraser, A.M., Reconstructing attractors for chaotic nonlinear time series: a comparison of singular system and redundancy criteria, Physica, 34D (1989) 391-404.

[21] Freeman, W.J., The physiology of perception, Sci. Am., 2 (1991) 78-95.

[22] Fuster, J.M., The Prefrontal Cortex, Raven, New York, 1980.

[23] Goldenberg, G., Podreka, I., Steiner, M. and Willmes, K., Patterns of regional cerebral blood flow related to memorizing of high and low imagery words: an emission computer tomography study, Neuropsychologia, 25 (1987) 473-485.

[24] Goldenberg, G., Podreka, I., Steiner, M., Willmes, K. et al., Regional cerebral blood flow patterns in visual imagery, $\mathrm{Neu}$ ropsychologia, 27 (1989) 641-664.

[25] Goldman-Rakic, P.S., Circuitry of primate prefrontal cortex and regulation of behavior by representational memory. In V.B.
Mountcastle, F. Plum and S.G. Geiger (Eds.), Handbook of Physiology, The Nervous System, 1987, pp. 373-417.

[26] Hebb, D.O., Concerning imagery, Psychol. Rev., 75 (1968) 466 477.

[27] Iasemidis, L.D., Sackellares, J.C., Zaveri, H.P. and William, W.J., Phase space topography and the Lyaponov exponent of electrocorticograms in partial seizures, Brain Topogr., 2 (1990) 187-201.

[28] Ingvar, D.H. and Philipsson, L., Distribution of cerebral blood flow in the dominant hemisphere during motor ideation and motor performance, Ann. Neurol., (1977) 230-237.

[29] Kosslyn, S.M., Ball, T.M. and Reiser, B.J., Visual images preserve metric spatial information: evidence from studies of image scanning, J. Exp. Psychol.: Human Percept. Perform., 4 (1978) 47-60.

[30] Lang, P.J., A bio-informational theory of emotional imagery, Psychophysiology, 16 (1979) 495-511.

[31] Lutzenberger, W., Birbaumer, N., Flor, H., Rockstroh, B. and Elbert, T., Dimensional analysis of the human EEG and intelligence, Neurosci. Lett., 143 (1992) 10-14.

[32] Lutzenberger, W., Elbert, T., Birbaumer, N., Ray, W.J. and Schupp, H., The scalp distribution of the fractal dimension of the EEG and its vriation with mental tasks, Brain Topogr., 5 (1992) 27-34.

[33] Lutzenberger, W. and Elbert, T., A/d conversion in DMA-mode - problems and solutions, Psychophysiology, 28 (1991) 607-608.

[34] Mulholland, T., The electro-encephalogram as an experimental tool in the study of internal attention gradients, Ann. NY Acad. Sci., 24 (1962) 664-669.

[35] Nan, $X$. and Jinghua, X., The fractal dimension of EEG as a physical measure of conscious human brain activities, Bull. Math. Biol., 50 (1988) 559-565.

[36] Palm, G., Cell assemblies as a guideline for brain research, Concepts Neurosci., 1 (1990) 133-147.

[37] Pijn, P.J., Van Nerven, J., Noest, A. and Lopez da Silva, F.H., Chaos or noise in EEG signals; dependence on state and brain site, Electroencephalogr. clin. Neurophysiol., 79 (1991) 371-381.

[38] Pritchard, W.S. and Duke, D.W., Human EEG using the Grassberger-Procaccia method, Psychophysiology, 29 (1992) 182-192.

[39] Rapp, P.E., Bashore, T.R., Martinerie, J.M., Albano, A.M., Zimmermann, I.D. and Mees, A.I., Dynamics of brain electrical activity, Brain Topogr., 2 (1989) 99-118.

[40] Ray, W.J. and Cole, H., EEG alpha reflects attentional demands, beta reflects emotional and cognitive processes, Science, 228 (1985) 750-752.

[41] Roland, P.E., Ericksson, S., Stone-Elander and Widen, L., Does mental activity change the oxidative metabolism of the brain?, Trends Neurosci., 7 (1987) 430-435.

[42] Roland, P.E. and Friberg, L., Localization of cortical areas activated by thinking, J. Neurophysiol., 53 (1985) 1219-1243.

[43] Sarbin, T.R., Imagining as muted role-taking: a historical-linguistic analysis. In P.W. Sheehan (Eds.), The Function and Nature of Imagery, Academic Press, New York, 1972, pp. 333-354.

[44] Shepard, R.N., Ecological constraints on internal representation: Resonant kinetics of perceiving, imagining, thinking, and dreaming, Psychol. Rev., 91 (1984) 417-447.

[45] Shepard, R.N., and Cooper, L.A., Mental images and their Transformations, MIT Press/Bradford Books, Cambridge, MA, 1982.

[46] Skarda, C.A. and Freeman, W.J., How brain makes chaos in order to make sense of the world, Behav. Brain Sci., 10 (1987) 161-195.

[47] Smith, L.A., Intrinsic limits on dimension calculations, Phys. Lett. A, 133 (1988) 283-288.

[48] Yeterian, E.H. and Pandya, D.N., Architectonic features of the primate brain: implications for information processing and behavior. In H.J. Markowitsch (Eds.), Information Processing by the Brain, Hans Huber, Toronto, 1988, pp. 7-38. 